\title{
Unified integral associated with the generalized V-function
}

\author{
S. Chandak', S.K.Q. Al-Omari² (D) and D.L. Suthar ${ }^{3}$
}

"Correspondence:

s.k.q.alomari@fet.edu.jo;

shridehalomari@bau.edu.jo

${ }^{2}$ Department of Physics and

Applied Sciences, Al-Balqa Applied University, Amman, Jordan

Full list of author information is

available at the end of the article

\begin{abstract}
In this paper, we present two new unified integral formulas involving a generalized $V$-function. Some interesting special cases of the main results are also considered in the form of corollaries. Due to the general nature of the $V$-function, several results involving different special functions such as the exponential function, the Mittag-Leffler function, the Lommel function, the Struve function, the Wright generalized Bessel function, the Bessel function and the generalized hypergeometric function are obtained by specializing the parameters in the presented formulas. More results are also discussed in detail.
\end{abstract}

MSC: Primary 26A33; 33B15; secondary 33C05; 44A10

Keywords: V-function; Oberhettinger's integral formula; Lavoie-Trottier integral formula

\section{Introduction and preliminaries}

Fractional calculus is as old as the conventional calculus and has been recently applied in various areas of engineering, science, finance, applied mathematics and bioengineering. The $\mathrm{V}$-function is an important special function that provides solutions to a number of problems formulated in terms of fractional order differential, integral and difference equations, therefore it has recently become a subject of interest for many authors in the field of fractional calculus and its applications. In addition, a number of researchers (see $[10,11,13,17,19,22,29,30])$ have studied in depth properties, applications and diverse extensions of a range of operators of fractional calculus, this field being very active and extensive around the world. One may refer to the research monographs [12] and [21] for further investigations in the area. Recently, the V-function is defined by Kumar [14] as follows:

$$
\begin{aligned}
V(z) & =V_{n}^{a_{u}, h, b_{v}}\left(l, \mu, \zeta, \delta, m, k_{u}, A_{v}, B_{w}, \eta, v, \rho ; z\right) \\
& =\xi \sum_{n=0}^{\infty} \frac{(-l)^{n} \prod_{u=1}^{p}\left[\left(a_{u}\right)_{n+k_{u}}\right](h+\eta n+v)^{-\mu}(z / 2)^{n \zeta+h \delta+m}}{\prod_{v=1}^{q}\left[\left(b_{v}\right)_{n+A_{v}}\right] \prod_{w=1}^{r}\left[(h)_{\eta n \rho+B_{w}}\right]},
\end{aligned}
$$

where

1. $l, \zeta, \delta, m, v, \rho, k_{u}(u=1, \ldots, p), A_{v}(v=1, \ldots, q), B_{w}(w=1, \ldots, r)$ are real numbers,

(c) The Author(s) 2020. This article is licensed under a Creative Commons Attribution 4.0 International License, which permits use, sharing, adaptation, distribution and reproduction in any medium or format, as long as you give appropriate credit to the original author(s) and the source, provide a link to the Creative Commons licence, and indicate if changes were made. The images or other third party material in this article are included in the article's Creative Commons licence, unless indicated otherwise in a credit line to the material. If material is not included in the article's Creative Commons licence and your intended use is not permitted by statutory regulation or exceeds the permitted use, you will need to obtain permission directly from the copyright holder. To view a copy of this licence, visit http://creativecommons.org/licenses/by/4.0/. 
2. $p, q$, and $r$ are natural numbers,

3. $a_{u}, b_{v} \geq 1(u=1, \ldots, p ; v=1, \ldots, q)$,

4. $\eta>0, \Re(\mu)>0, \Re(h)>0, z$ is a complex variable and $\xi$ is an arbitrary constant,

5. the series on the RHS of (1.1) converges absolutely if $p<q$ or $p=q$ with $\left|l(z / 2)^{\zeta}\right| \leq 1$.

For further information on the constraints of the convergence of the RHS of the series (1.1), we refer to Refs. $[15,16]$. The V-function defined by (1.1) is of general character as it assimilates a variety of valuable functions such as the Macrobert E-function, the exponential function [4], the generalized Mittag-Leffler function [9, 23, 31, 34], the Lommel function [8], the Struve function [26, 33, 35], the generalized Bessel function [27], the Bessel function $[5,36]$, the generalized hypergeometric function $[4,32,37,38]$ and the unified Riemann-Zeta function [7].

Some special cases of the $\mathrm{V}$-function (1.1) are as follows:

(i) For $w=1, h=1, p=P, q=Q, l=-2, \mu=1, \zeta=1, \delta=0, m=0, k_{u}=0, A_{v}=0, B_{1}=$ $-1, \eta=1, v=-1, \rho=1$ and $\xi=1$, the $\mathrm{V}$-function (1.1) turns into a generalized hypergeometric function (see, e.g., [4]),

$$
V_{n}^{a_{u}, 1, b_{v}}(-2,1,1,0,0,0,0,-1,1,-1,1 ; z)={ }_{P} F_{Q}\left(a_{P} ; b_{Q}, z\right) .
$$

(ii) For $u=1, v=2, w=1, a_{1}=1, b_{1}=1, b_{2}=1, l=1, \mu=1, \zeta=2, \delta=1, m=0, k_{1}=$ $0, A_{1}=0, A_{2}=0, B_{1}=0, \eta=1, v=0, \rho=1$ and $\xi=1 /(\Gamma(h))$, the $V$-function (1.1) turns into a Bessel function (see, e.g., [5]),

$$
V_{n}^{1, h, 1,1}(1,1,2,1,0,0,0,0,0,1,0,1 ; z)=J_{h}(z)
$$

(iii) For $u=1, v=2, w=1, a_{1}=1, b_{1}=1, b_{2}=1, l=2, \mu=1, \zeta=1, \delta=1, m=0, k_{1}=$ $0, A_{1}=0, A_{2}=0, B_{1}=0, v=0, \rho=1$ and $\xi=1 /(\Gamma(h))$, the $\mathrm{V}$-function (1.1) turns into the Wright generalized Bessel function (see, e.g., [5]),

$$
V_{n}^{1, h, 1,1}(1,1,2,1,0,0,0,0,0, \eta, 0,1 ; z)=J_{h}^{\eta}(z) .
$$

(iv) For $u=1, v=2, w=1, a_{1}=1, b_{1}=3 / 2, b_{2}=1, l=1, \mu=1, \zeta=2, \delta=1, m=1, k_{1}=$ $0, A_{1}=0, A_{2}=0, B_{1}=1 / 2, \eta=1, v=1 / 2, \rho=1$ and $\xi=1 / \Gamma(h) \Gamma(3 / 2)$, the V-function (1.1) turns into the Struve function (see, e.g., [5]),

$$
V_{n}^{1, h, 3 / 2,1}(1,1,2,1,1,0,0,0,1 / 2,1,1 / 2,1 ; z)=H_{h}(z) .
$$

(v) For $u=1, v=2, w=1, a_{1}=1, b_{1}=(\tau+\epsilon+3) / 2, b_{2}=(\tau-\epsilon+3) / 2, l=1, \mu=1, \zeta=$ $2, h=1, \delta=\tau, m=1, k_{1}=0, A_{1}=0, A_{2}=0, B_{1}=-1, \eta=1, \nu=-1, \rho=1$ and $\xi=2^{\tau+1} /\{(\tau+\epsilon+1)(\tau-\epsilon+1)\}$, the V-function (1.1) turns into the Lommel function (see, e.g., [5]),

$$
V_{n}^{1,1,(\tau+\epsilon+3) / 2,(\tau-\epsilon+3) / 2}(1,1,2, \tau, 1,0,0,0,-1,1,1,1 ; z)=S_{\tau, \epsilon}(z) .
$$

(vi) For $u=1, v=1, w=1, a_{1}=1, b_{1}=1, l=-2, \mu=1, \zeta=1, \delta=0, m=0, k_{1}=0, A_{1}=$ $0, B_{1}=-1, v=-1, \rho=1$ and $\xi=1 /(\Gamma(h))$, the V-function (1.1) turns into the Mittag-Leffler function (see, e.g., $[9,23]$ ),

$$
V_{n}^{1, h, 1}(-2,1,1,0,0,0,0,-1, \eta,-1,1 ; z)=E_{\eta, h}(z) .
$$


(vii) For $u=1, v=1, w=1, a_{1}=a, b_{1}=1, l=-2, \zeta=1, \delta=0, m=0, k_{1}=0, A_{1}=0, B_{1}=$ $0, \eta=1 v=0, \rho=0$ and $\xi=1$, the $\mathrm{V}$-function (1.1) turns into the unified Riemann-Zeta function (see, e.g., [7]),

$$
V_{n}^{a, h, 1}(-2, \mu, 1,0,0,0,0,0,1,0,0 ; z)=\phi_{a}(z, \mu, h)
$$

(viii) For $u=1, v=1, w=1, a_{1}=1, b_{1}=1, l=2, \zeta=1, \delta=0, m=0, k_{1}=0, A_{1}=0, B_{1}=$ $-1, \eta=1 v=-1, \rho=1, h=1, \mu=1$, and $\xi=1$, the V-function (1.1) turns into the $e^{-z}$ function,

$$
V_{n}^{1,1,1}(2,1,1,0,0,0,0,-1,1,-1,1 ; z)=e^{-z}
$$

(ix) For $w=1, p=P, q=Q, l=2, \mu=1, \zeta=1, \delta=0, m=0, k_{u}=0, A_{u}=0, B_{1}=-1, \eta=$ $1 v=-1, \rho=1, h=1$ and $\xi=\frac{\prod_{u=1}^{P} \Gamma\left(a_{u}\right)}{\prod_{v=1}^{Q} \Gamma\left(b_{v}\right)}$, the V-function (1.1) turns into the Macrobert E-function (see, e.g., [4]),

$$
V_{n}^{1,1,1}(2,1,1,0,0,1,0,-1,1,-1,1 ; z)=E\left[P ;\left(a_{P}\right) ; Q ;\left(b_{Q}\right) ; z^{-1}\right] .
$$

(x) For $u=1, v=2, w=1, a_{1}=1, b_{1}=1, k_{1}=0, h=1 / 2, l=1, \mu=1, \zeta=2, \delta=0, m=$ $0, A_{1}=0, A_{2}=-1, B_{1}=0, \eta=1 v=-1 / 2, \rho=1$ and $\xi=1$, the $\mathrm{V}$-function (1.1) turns into the $\cos z$ function,

$$
V_{n}^{1,1 / 2,1,1}(1,1,2,0,0,0,0,-1,0,1,-1 / 2,1 ; z)=\cos z
$$

(xi) For $u=1, v=2, w=1, a_{1}=1, b_{1}=1, k_{1}=0, h=1 / 2, l=1, \mu=1, \zeta=2, \delta=2, m=$ $0, A_{1}=0, A_{2}=-1, B_{1}=0, \eta=1 v=-1 / 2, \rho=1$ and $\xi=1$, the $\mathrm{V}$-function (1.1) turns into the $\sin z$ function,

$$
V_{n}^{1,1 / 2,1,1}(1,1,2,2,0,0,0,-1,0,1,-1 / 2,1 ; z)=\sin z
$$

To proceed in our next investigation, we need to recall the following Oberhettinger integral formula [28]:

$$
\int_{0}^{\infty} z^{\lambda-1}\left(z+b+\sqrt{z^{2}+2 b z}\right)^{-\gamma} d z=2 \gamma b^{-\lambda}\left(\frac{b}{2}\right)^{\lambda} \frac{\Gamma(2 \lambda) \Gamma(\gamma-\lambda)}{\Gamma(1+\lambda+\gamma)}
$$

provided that $0<\Re(\lambda)<\Re(\gamma)$. Also, we need to recall the Lavoie-Trottier integral formula [18]:

$$
\int_{0}^{1} z^{\lambda-1}(1-z)^{2 \gamma-1}\left(1-\frac{z}{3}\right)^{2 \lambda-1}\left(1-\frac{z}{4}\right)^{\gamma-1} d z=\left(\frac{2}{3}\right)^{2 \gamma} \frac{\Gamma(\gamma) \Gamma(\lambda)}{\Gamma(\lambda+\gamma)}
$$

provided that $\Re(\lambda)>0, \Re(\gamma)>0$. For further investigations of the function, the reader may be referred to the recent work of $[1-3,20,24,25]$ and the references therein.

However, the main object of this paper is to establish certain new integrals involving the $\mathrm{V}$-function. The results are presented as theorems and corollaries that may potentially be very useful. At last, we establish special cases of our main results connecting various special functions. 


\section{Main results}

In this section, we establish four generalized integral formulas for the V-function. These formulas are given by the following theorems.

Theorem 2.1 Let $\lambda, \gamma, \alpha \in \mathbb{C} ; l, \zeta, \delta, m, v, \rho, k_{u}, A_{v}, B_{w} \in \Re ; b, p, q, r \in \mathbb{N} ; a_{u}, b_{v} \geq 1, \eta>$ $0, \Re(\mu)>0, \Re(h)>0, \Re(\alpha)>0, z>0$ and $\xi>0$ be arbitrary constants, such that $0<\Re(\lambda)<$ $\Re(\gamma+\alpha(n \zeta+h \delta+m))$. Then the following integral holds true:

$$
\begin{aligned}
\int_{0}^{\infty} & z^{\lambda-1} A^{-\gamma} V_{n}^{a_{u}, h, b_{v}}\left(l, \mu, \zeta, \delta, m, k_{u}, A_{v}, B_{w}, \eta, v, \rho ; \frac{y}{A^{\alpha}}\right) d z \\
= & 2^{1-\lambda} b^{\lambda-\gamma} \Gamma(2 \lambda) \xi \sum_{n=0}^{\infty} \frac{(-l)^{n} \prod_{u=1}^{p}\left[\left(a_{u}\right)_{n+k_{u}}\right](h+\eta n+v)^{-\mu}\left(y / 2 b^{\alpha}\right)^{n \zeta+h \delta+m}}{\prod_{v=1}^{q}\left[\left(b_{v}\right)_{n+A_{v}}\right] \prod_{w=1}^{r}\left[(h)_{\eta n \rho+B_{w}}\right]} \\
& \times \frac{\Gamma(\gamma+\alpha(n \zeta+h \delta+m)+1) \Gamma(\gamma-\lambda+\alpha(n \zeta+h \delta+m))}{\Gamma(\gamma+\lambda+\alpha(n \zeta+h \delta+m)+1) \Gamma(\gamma+\alpha(n \zeta+h \delta+m))}
\end{aligned}
$$

where $A=\left(z+b+\sqrt{z^{2}+2 b z}\right)$.

Proof For the convenience of the reader, we denote the left-hand side of (2.1) by $\mathfrak{\Im}_{1}$. Therefore, by invoking (1.1) in the integrand (2.1) and interchanging the order of integration and summation, which is verified by the uniform convergence of the involved series under the given conditions, we get

$$
\begin{aligned}
\Im_{1}= & \xi \sum_{n=0}^{\infty} \frac{(-l)^{n} \prod_{u=1}^{p}\left[\left(a_{u}\right)_{n+k_{u}}\right](h+\eta n+v)^{-\mu}(y / 2)^{n \zeta+h \delta+m}}{\prod_{v=1}^{q}\left[\left(b_{v}\right)_{n+A_{v}}\right] \prod_{w=1}^{r}\left[(h)_{\eta n \rho+B_{w}}\right]} \\
& \times \int_{0}^{\infty} z^{\lambda-1} A^{-(\gamma+\alpha(n \zeta+h \delta+m))} d z .
\end{aligned}
$$

Hence, on applying the integral formula (1.13) for the integral in (2.2), we, under the valid conditions, obtain the following expression:

$$
\begin{aligned}
\Im_{1}= & \xi \sum_{n=0}^{\infty} \frac{(-l)^{n} \prod_{u=1}^{p}\left[\left(a_{u}\right)_{n+k_{u}}\right](h+\eta n+v)^{-\mu}(y / 2)^{n \zeta+h \delta+m}}{\prod_{v=1}^{q}\left[\left(b_{v}\right)_{n+A_{v}}\right] \prod_{w=1}^{r}\left[(h)_{\eta n \rho+B_{w}}\right]} \\
& \times 2(\gamma+\alpha(n \zeta+h \delta+m))\left(\frac{b}{2}\right)^{\lambda} b^{(-(\gamma+\alpha(n \zeta+h \delta+m))} \\
& \times \frac{\Gamma(2 \lambda) \Gamma(\gamma+\alpha(n \zeta+h \delta+m)-\lambda)}{\Gamma(1+\gamma+\alpha(n \zeta+h \delta+m)+\lambda)} \\
= & 2^{1-\lambda} b^{\lambda-\gamma} \xi \sum_{n=0}^{\infty} \frac{(-l)^{n} \prod_{u=1}^{p}\left[\left(a_{u}\right)_{n+k_{u}}\right](h+\eta n+v)^{-\mu}\left(y / 2 b^{\alpha}\right)^{n \zeta+h \delta+m}}{\prod_{v=1}^{q}\left[\left(b_{v}\right)_{n+A_{v}}\right] \prod_{w=1}^{r}\left[(h)_{\eta n \rho+B_{w}}\right]} \\
& \times \frac{\Gamma(2 \lambda) \Gamma(1+\gamma+\alpha(n \zeta+h \delta+m)) \Gamma(\gamma+\alpha(n \zeta+h \delta+m)-\lambda)}{\Gamma(\gamma+\alpha(n \zeta+h \delta+m)) \Gamma(1+\gamma+\alpha(n \zeta+h \delta+m)+\lambda)}
\end{aligned}
$$

which is the desired result.

Theorem 2.2 Let $\lambda, \gamma, \alpha \in \mathbb{C} ; l, \zeta, \delta, m, v, \rho, k_{u}, A_{v}, B_{w} \in \Re ; b, p, q, r \in \mathbb{N} ; a_{u}, b_{v} \geq 1, \eta>$ $0, \Re(\mu)>0, \Re(h)>0, \Re(\alpha)>0, z>0$ and $\xi>0$ be arbitrary constants, such that $0<\Re(\lambda)<$ 
$\Re(\gamma+\alpha(n \zeta+h \delta+m))$. Then the following integral holds true:

$$
\begin{aligned}
\int_{0}^{\infty} & z^{\lambda-1} A^{-\gamma} V_{n}^{a_{u}, h, b_{v}}\left(l, \mu, \zeta, \delta, m, k_{u}, A_{v}, B_{w}, \eta, v, \rho ; \frac{y z^{\alpha}}{A^{\alpha}}\right) d z \\
= & 2^{1-\lambda} b^{\lambda-\gamma} \Gamma(\gamma-\lambda) \xi \sum_{n=0}^{\infty} \frac{(-l)^{n} \prod_{u=1}^{p}\left[\left(a_{u}\right)_{n+k_{u}}\right](h+\eta n+v)^{-\mu}}{\prod_{v=1}^{q}\left[\left(b_{v}\right)_{n+A_{v}}\right] \prod_{w=1}^{r}\left[(h)_{\eta n \rho+B_{w}}\right]} \\
& \times\left(\frac{y}{2^{\alpha+1}}\right)^{n \zeta+h \delta+m} \frac{\Gamma(\gamma+\alpha(n \zeta+h \delta+m)+1) \Gamma(2 \lambda+2 \alpha(n \zeta+h \delta+m))}{\Gamma(\gamma+\lambda+2 \alpha(n \zeta+h \delta+m)+1) \Gamma(\gamma+\alpha(n \zeta+h \delta+m))}
\end{aligned}
$$

Proof By following a technique similar to what has already been used in the proof of Theorem 2.1 , we can easily prove the integral formula (2.3). Therefore, we omit the detailed proof.

Theorem 2.3 Let $\lambda, \gamma, \alpha \in \mathbb{C} ; l, \zeta, \delta, m, v, \rho, k_{u}, A_{v}, B_{w} \in \Re ; p, q, r \in \mathbb{N} ; a_{u}, b_{v} \geq 1, \eta>$ $0, \Re(\mu)>0, \Re(h)>0, \Re(\alpha)>0, z>0$ and $\xi>0$ be arbitrary constants, such that $0<\Re(\lambda)<$ $\mathfrak{R}(\gamma+\alpha(n \zeta+h \delta+m))$. Then the following integral holds true:

$$
\begin{aligned}
\int_{0}^{1} z^{\lambda-1}(1-z)^{2 \gamma-1} S^{2 \lambda-1} T^{\gamma-1} \\
\quad \times V_{n}^{a_{u}, h, b_{v}}\left(l, \mu, \zeta, \delta, m, k_{u}, A_{v}, B_{w}, \eta, v, \rho ; y(1-z)^{2} T^{\alpha}\right) d z \\
=\left(\frac{2}{3}\right)^{2 \lambda} \Gamma(\lambda) \xi \sum_{n=0}^{\infty} \frac{(-l)^{n} \prod_{u=1}^{p}\left[\left(a_{u}\right)_{n+k_{u}}\right](h+\eta n+\nu)^{-\mu}(y / 2)^{n \zeta+h \delta+m}}{\prod_{v=1}^{q}\left[\left(b_{v}\right)_{n+A_{v}}\right] \prod_{w=1}^{r}\left[(h)_{\eta n \rho+B_{w}}\right]} \\
\quad \times \frac{\Gamma(\gamma+\alpha(n \zeta+h \delta+m))}{\Gamma(\lambda+\gamma+\alpha(n \zeta+h \delta+m))},
\end{aligned}
$$

where $S=\left(1-\frac{z}{3}\right)$ and $T=\left(1-\frac{z}{4}\right)$.

Proof For more convenience, we denote the left-hand side of (2.4) by $\mathfrak{\Im}_{2}$. Therefore, by invoking (1.1) in the integral part of (2.4) and interchanging the order of integration and summation, which is verified by uniform convergence of the involved series under the given conditions, we obtain

$$
\begin{aligned}
\Im_{2}= & \xi \sum_{n=0}^{\infty} \frac{(-l)^{n} \prod_{u=1}^{p}\left[\left(a_{u}\right)_{n+k_{u}}+\right](h+\eta n+v)^{-\mu}(y / 2)^{n \zeta+h \delta+m}}{\prod_{\nu=1}^{q}\left[\left(b_{v}\right)_{n+A_{v}}\right] \prod_{w=1}^{r}\left[(h)_{\eta n \rho+B_{w}}\right]} \\
& \times \int_{0}^{1} z^{\lambda-1}(1-z)^{2 \gamma+2 \alpha(n \zeta+h \delta+m)-1}(S)^{2 \lambda-1}(T)^{\gamma+\alpha(n \zeta+h \delta+m)-1} d z .
\end{aligned}
$$

Now, upon applying the integral formula (1.14) to the integral part of (2.5) we obtain the following expression under their valid conditions:

$$
\begin{aligned}
\Im_{2}= & \xi \sum_{n=0}^{\infty} \frac{(-l)^{n} \prod_{u=1}^{p}\left[\left(a_{u}\right)_{n+k_{u}}\right](h+\eta n+v)^{-\mu}(y / 2)^{n \zeta+h \delta+m}}{\prod_{v=1}^{q}\left[\left(b_{v}\right)_{n+A_{v}}\right] \prod_{w=1}^{r}\left[(h)_{\eta n \rho+B_{w}}\right]} \\
& \times\left(\frac{2}{3}\right)^{2 \lambda} \frac{\Gamma(\lambda) \Gamma(\gamma+\alpha(n \zeta+h \delta+m))}{\Gamma(\lambda+\gamma+\alpha(n \zeta+h \delta+m))}
\end{aligned}
$$




$$
\begin{aligned}
= & \left(\frac{2}{3}\right)^{2 \lambda} \Gamma(\lambda) \xi \sum_{n=0}^{\infty} \frac{(-l)^{n} \prod_{u=1}^{p}\left[\left(a_{u}\right)_{n+k_{u}}\right](h+\eta n+v)^{-\mu}(y / 2)^{n \zeta+h \delta+m}}{\prod_{v=1}^{q}\left[\left(b_{v}\right)_{n+A_{v}}\right] \prod_{w=1}^{r}\left[(h)_{\eta n \rho+B_{w}}\right]} \\
& \times \frac{\Gamma(\gamma+\alpha(n \zeta+h \delta+m))}{\Gamma(\lambda+\gamma+\alpha(n \zeta+h \delta+m))},
\end{aligned}
$$

which is the desired result.

Theorem 2.4 Let $\lambda, \gamma, \alpha \in \mathbb{C} ; l, \zeta, \delta, m, v, \rho, k_{u}, A_{v}, B_{w} \in \Re ; p, q, r \in \mathbb{N} ; a_{u}, b_{v} \geq 1, \eta>$ $0, \Re(\mu)>0, \Re(h)>0, \Re(\alpha)>0, z>0$ and $\xi>0$ be arbitrary constants, such that $0<\Re(\lambda)<$ $\Re(\gamma+\alpha(n \zeta+h \delta+m))$. Then the following integral holds true:

$$
\begin{gathered}
\int_{0}^{1} z^{\lambda-1}(1-z)^{2 \gamma-1} S^{2 \lambda-1} T^{\gamma-1} V_{n}^{a_{u}, h, b_{v}}\left(l, \mu, \zeta, \delta, m, k_{u}, A_{v}, B_{w}, \eta, v, \rho ; y z^{\alpha} S^{2 \alpha}\right) d z \\
=\left(\frac{2}{3}\right)^{2 \lambda} \Gamma(\gamma) \xi \sum_{n=0}^{\infty} \frac{(-l)^{n} \prod_{u=1}^{p}\left[\left(a_{u}\right)_{n+k_{u}}\right](h+\eta n+v)^{-\mu}(y / 2)^{n \zeta+h \delta+m}}{\prod_{v=1}^{q}\left[\left(b_{v}\right)_{n+A_{v}}\right] \prod_{w=1}^{r}\left[(h)_{\eta n \rho+B_{w}}\right]} \\
\quad \times\left(\frac{2}{3}\right)^{2 \alpha(n \zeta+h \delta+m)} \frac{\Gamma(\lambda+\alpha(n \zeta+h \delta+m))}{\Gamma(\lambda+\gamma+\alpha(n \zeta+h \delta+m))} .
\end{gathered}
$$

Proof By following the proof of Theorem 2.3, step by step, Eq. (2.6) can easily be obtained. Hence, we omit the detailed proof.

\section{Special cases}

In this section, we aim to present some special cases by adopting certain advisable values of the parameters imposed on Theorems 2.1, 2.2, 2.3 and 2.4. Indeed, such constraints led up to certain interesting results concerning generalized hypergeometric functions, Bessel functions, Wright generalized Bessel functions, Struve functions, Lommel functions, Mittag-Leffler functions, Riemann-Zeta functions, exponential functions and Macrobert E-functions as follows.

(i) By inserting $w=1, h=1, p=P, q=Q, l=-2, \mu=1, \zeta=1, \delta=0, m=0, k_{u}=0, A_{v}=$ $0, B_{1}=-1, \eta=1, v=-1, \rho=1$ and $\xi=1$ in Eqs. (2.1), (2.3), (2.4) and (2.6), the V-function turns into the generalized hypergeometric function [4]. Moreover, by applying the result ([6], Eq. (2.1.2.2), pp. 34)

$$
(\beta)_{\alpha n}=\alpha^{\alpha n} \prod_{i=1}^{\alpha}\left[\frac{\beta+i-1}{\alpha}\right]_{n}
$$

under the assumption that the conditions of Theorems 2.1-2.4 are employed, we, respectively, get

$$
\begin{aligned}
\int_{0}^{\infty} z^{\lambda-1} A^{-\gamma}{ }_{P} F_{Q}\left(a_{P} ; b_{Q} ; \frac{y}{A^{\alpha}}\right) d z \\
=2^{1-\lambda} b^{\lambda-\gamma} \gamma \frac{\Gamma(2 \lambda) \Gamma(\gamma-\lambda)}{\Gamma(\lambda+\gamma+1)} \\
\quad \times{ }_{P+2 \alpha} F_{Q+2 \alpha}\left[\begin{array}{l}
a_{1}, a_{2}, \ldots, a_{P} ; \frac{1+\gamma}{\alpha}, \frac{2+\gamma}{\alpha} \ldots, \frac{\gamma+\alpha}{\alpha}, \\
b_{1}, b_{2}, \ldots, b_{Q} ; \frac{\gamma}{\alpha}, \frac{1+\gamma}{\alpha} \cdots, \frac{\gamma+\alpha-1}{\alpha},
\end{array}\right.
\end{aligned}
$$




$$
\begin{aligned}
& \left.\begin{array}{l}
\left.\frac{\gamma-\lambda}{\alpha}, \frac{\gamma-\lambda+1}{\alpha} \cdots, \frac{\gamma-\lambda+\alpha-1}{\alpha} \mid y b^{-\alpha}\right], \\
\frac{\lambda+\gamma+1}{\alpha}, \frac{\lambda+\gamma+2}{\alpha} \cdots, \frac{\lambda+\gamma+\alpha}{\alpha}
\end{array}\right] \\
& \int_{0}^{\infty} z^{\lambda-1} A^{-\gamma}{ }_{P} F_{Q}\left(a_{P} ; b_{Q} ; \frac{y z^{\alpha}}{A^{\alpha}}\right) d z \\
& =2^{1-\lambda} b^{\lambda-\gamma} \gamma \frac{\Gamma(2 \lambda) \Gamma(\gamma-\lambda)}{\Gamma(\lambda+\gamma+1)} \\
& \times_{P+3 \alpha} F_{Q+3 \alpha}\left[\begin{array}{l}
a_{1}, a_{2}, \ldots, a_{P} ; \frac{1+\gamma}{\alpha}, \frac{2+\gamma}{\alpha} \cdots, \frac{\gamma+\alpha}{\alpha} \\
b_{1}, b_{2}, \ldots, b_{Q} ; \frac{\gamma}{\alpha}, \frac{1+\gamma}{\alpha} \cdots, \frac{\gamma+\alpha-1}{\alpha}
\end{array}\right. \\
& \left.\frac{2 \lambda}{2 \alpha}, \frac{2 \lambda+1}{2 \alpha} \cdots, \frac{2 \lambda+2 \alpha-1}{2 \alpha}\left|\frac{\lambda+\gamma+1}{2 \alpha}, \frac{\lambda+\gamma+2}{2 \alpha} \cdots, \frac{\lambda+\gamma+2 \alpha}{2 \alpha}\right| 2^{-\alpha} y\right] \text {, } \\
& \int_{0}^{1} z^{\lambda-1}(1-z)^{2 \gamma-1}(S)^{2 \lambda-1}(T)^{\gamma-1}{ }_{P} F_{Q}\left(a_{P} ; b_{Q} ; y(1-z)^{2 \alpha} T^{\alpha}\right) d z
\end{aligned}
$$

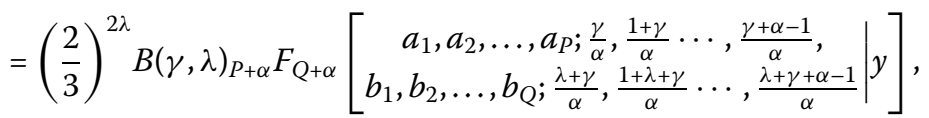

$$
\begin{aligned}
& \int_{0}^{1} z^{\lambda-1}(1-z)^{2 \gamma-1}(S)^{2 \lambda-1}(T)^{\gamma-1}{ }_{P} F_{Q}\left(a_{P} ; b_{Q} ; y(z)^{\alpha} S^{\alpha}\right) d z
\end{aligned}
$$

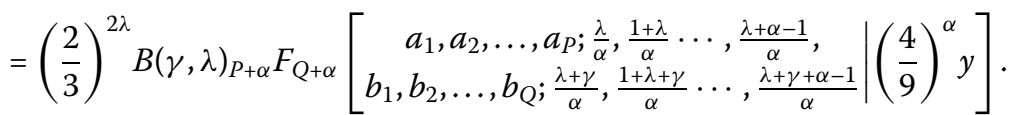

(ii) Putting $\alpha=1, u=1, v=2, w=1, a_{1}=1, b_{1}=1, b_{2}=1, l=1, \mu=1, \zeta=2, \delta=1, m=$ $0, k_{1}=0, A_{1}=0, A_{2}=0, B_{1}=0, \eta=1, v=0, \rho=1$ and $\xi=1 /(\Gamma(h))$ in Eqs. (2.1), (2.3), (2.4) and (2.6), the V-function turns to a Bessel function [5]. By applying the result from ([6], Eq. (2.1.5.3), pp. 38)

$$
(\beta)_{2 n}=2^{2 n}\left(\frac{\beta}{2}\right)_{n}\left(\frac{\beta+1}{2}\right)_{n}
$$

when the conditions already imposed on Theorems 2.1-2.4 are applied, we, respectively, have

$$
\begin{aligned}
& \int_{0}^{\infty} z^{\lambda-1} A^{-\gamma} J_{h}\left(\frac{y}{A}\right) d z=2^{1-\lambda-h} b^{\lambda-h-\gamma}(\gamma+h) y^{h} \\
& \times \frac{\Gamma(2 \lambda) \Gamma(\gamma+h-\lambda)}{\Gamma(\lambda+\gamma+h+1) \Gamma(1+h)}
\end{aligned}
$$

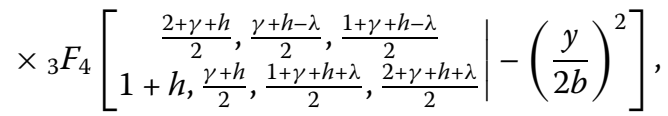

$$
\begin{aligned}
& \int_{0}^{\infty} z^{\lambda-1} A^{-\gamma} J_{h}\left(\frac{y z}{A}\right) d z=2^{-\gamma-2 h} b^{\lambda-\gamma} y^{h}(\gamma+h) \\
& \times \frac{\Gamma(\gamma-h) \Gamma(\lambda+h) \Gamma\left(\frac{2 \lambda+2 h+1}{2}\right)}{\Gamma(h+1)(\lambda+\gamma+1) \Gamma\left(\frac{\lambda+\gamma+2 h+1}{2}\right) \Gamma\left(\frac{\lambda+\gamma+2 h+2}{2}\right)}
\end{aligned}
$$

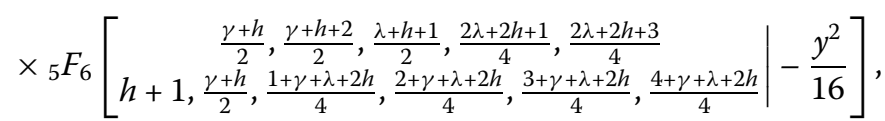




$$
\begin{aligned}
& \int_{0}^{1} z^{\lambda-1}(1-z)^{2 \gamma-1}(S)^{2 \lambda-1}(T)^{\gamma-1} J_{h}\left(y(1-z)^{2} T\right) d z
\end{aligned}
$$

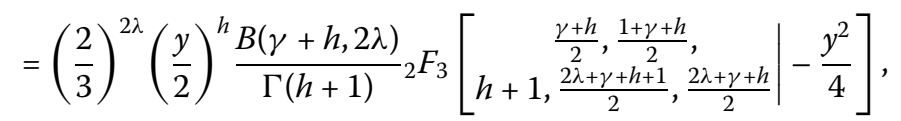

$$
\begin{aligned}
& \int_{0}^{1} z^{\lambda-1}(1-z)^{2 \gamma-1}(S)^{2 \lambda-1}(T)^{\gamma-1} J_{h}\left(y z S^{2}\right) d z
\end{aligned}
$$

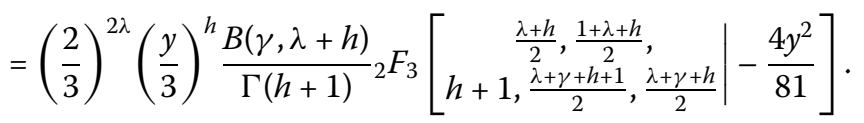

(iii) Putting $u=1, v=2, w=1, a_{1}=1, b_{1}=1, b_{2}=1, l=2, \mu=1, \zeta=1, \delta=1, m=0, k_{1}=$ $0, A_{1}=0, A_{2}=0, B_{1}=0, v=0, \rho=1$ and $\xi=1 /(\Gamma(h))$ in Eqs. (2.1), (2.3), (2.4) and (2.6), the V-function turns to a Wright generalized Bessel function [5]. Similarly, upon using the result (3.1) under the assumption that the conditions in Theorems 2.1-2.4 are applied, we, respectively, get

$$
\begin{aligned}
& \int_{0}^{\infty} z^{\lambda-1} A^{-\gamma} J_{h}^{\eta}\left(\frac{y}{A^{\alpha}}\right) d z \\
& =2^{1-\lambda} b^{\lambda-\gamma} \gamma \frac{\Gamma(2 \lambda) \Gamma(\gamma-\lambda)}{\Gamma(h+1) \Gamma(\lambda+\gamma+1)} \\
& \times{ }_{2 \alpha} F_{2 \alpha+\eta}\left[\begin{array}{c}
\frac{1+\gamma}{\alpha}, \frac{2+\gamma}{\alpha} \cdots, \frac{\gamma+\alpha}{\alpha}, \\
\frac{h+1}{\eta}, \frac{h+2}{\eta}, \ldots, \frac{h+\eta}{\eta}, \frac{\gamma}{\alpha}, \frac{1+\gamma}{\alpha} \cdots, \frac{\gamma+\alpha-1}{\alpha},
\end{array}\right. \\
& \left.\begin{array}{l}
\frac{\gamma-\lambda}{\alpha}, \frac{\gamma-\lambda+1}{\alpha} \cdots, \frac{\gamma-\lambda+\alpha-1}{\alpha} \\
\frac{\lambda+\gamma+1}{\alpha}, \frac{\lambda+\gamma+2}{\alpha} \cdots, \frac{\lambda+\gamma+\alpha}{\alpha}
\end{array} \mid-\frac{y}{b^{\alpha} \eta^{\eta}}\right] \\
& \int_{0}^{\infty} z^{\lambda-1} A^{-\gamma} J_{h}^{\eta}\left(\frac{y z^{\alpha}}{A^{\alpha}}\right) d z \\
& =2^{1-\lambda} b^{\lambda-\gamma} \gamma \frac{\Gamma(2 \lambda) \Gamma(\gamma-\lambda)}{\Gamma(h+1) \Gamma(\lambda+\gamma+1)} \\
& \times{ }_{3 \alpha} F_{3 \alpha+\eta}\left[\begin{array}{c}
\frac{1+\gamma}{\alpha}, \frac{2+\gamma}{\alpha} \ldots, \frac{\gamma+\alpha}{\alpha}, \\
\frac{h+1}{\eta}, \frac{h+2}{\eta}, \ldots, \frac{h+\eta}{\eta}, \frac{\gamma}{\alpha}, \frac{1+\gamma}{\alpha} \cdots, \frac{\gamma+\alpha-1}{\alpha},
\end{array}\right.
\end{aligned}
$$

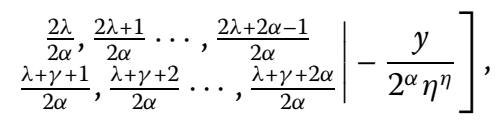

$$
\begin{aligned}
& \int_{0}^{1} z^{\lambda-1}(1-z)^{2 \gamma-1}(S)^{2 \lambda-1}(T)^{\gamma-1} J_{h}^{\eta}\left(y(1-z)^{2 \alpha} T^{\alpha}\right) d z \\
& =\left(\frac{2}{3}\right)^{2 \lambda} \frac{B(\gamma, \lambda)}{\Gamma(h+1)} \\
& \times{ }_{\alpha} F_{\alpha+\eta}\left[\begin{array}{c}
\frac{\gamma}{\alpha}, \frac{1+\gamma}{\alpha} \ldots, \frac{\gamma+\alpha-1}{\alpha}, \\
\left.\frac{h+1}{\eta}, \frac{h+2}{\eta}, \ldots, \frac{h+\eta}{\eta}, \frac{\lambda+\gamma}{\alpha}, \frac{1+\lambda+\gamma}{\alpha} \ldots, \frac{\lambda+\gamma+\alpha-1}{\alpha} \mid-\frac{y}{\eta^{\eta}}\right],
\end{array}\right. \\
& \int_{0}^{1} z^{\lambda-1}(1-z)^{2 \gamma-1}(S)^{2 \lambda-1}(T)^{\gamma-1} J_{h}^{\eta}\left(y z^{\alpha} S^{2 \alpha}\right) d z \\
& =\left(\frac{2}{3}\right)^{2 \lambda} \frac{B(\gamma, \lambda)}{\Gamma(h+1)}
\end{aligned}
$$




$$
\times{ }_{\alpha} F_{\alpha+\eta}\left[\begin{array}{c}
\frac{\lambda}{\alpha}, \frac{1+\lambda}{\alpha} \cdots, \frac{\lambda+\alpha-1}{\alpha}, \\
\left.\frac{h+1}{\eta}, \frac{h+2}{\eta}, \ldots, \frac{h+\eta}{\eta}, \frac{\lambda+\gamma}{\alpha}, \frac{1+\lambda+\gamma}{\alpha} \cdots, \frac{\lambda+\gamma+\alpha-1}{\alpha} \mid-\left(\frac{4}{9}\right)^{\alpha} \frac{y}{\eta^{\eta}}\right] .
\end{array}\right.
$$

(iv) By inserting $u=1, v=2, w=1, a_{1}=1, b_{1}=3 / 2, b_{2}=1, l=1, \mu=1, \zeta=2, \delta=1, m=$ $1, k_{1}=0, A_{1}=0, A_{2}=0, B_{1}=1 / 2, \eta=1, v=1 / 2, \rho=1$ and $\xi=1 / \Gamma(h) \Gamma(3 / 2)$ in Eqs. (2.1), (2.3), (2.4) and (2.6), the $\mathrm{V}$-function turns to a Struve function [5] and, by using the result (3.1) under the assumptions of Theorems 2.1-2.4, we, respectively, arrive at

$$
\begin{aligned}
& \int_{0}^{\infty} z^{\lambda-1} A^{-\gamma} H_{h}\left(\frac{y}{A^{\alpha}}\right) d z \\
& =2^{1-\lambda} b^{\lambda-(\gamma+\alpha(h+1))}(\gamma+\alpha(h+1))\left(\frac{y}{2}\right)^{h+1} \\
& \times \frac{\Gamma(2 \lambda) \Gamma(\gamma-\lambda+\alpha(h+1))}{\Gamma(1+\lambda+\gamma+\alpha(h+1)) \Gamma(3 / 2+h) \Gamma(3 / 2)} \\
& \times{ }_{1+4 \alpha} F_{2+4 \alpha}\left[\begin{array}{c}
1, \frac{1+\gamma+\alpha(h+1)}{2 \alpha}, \frac{2+\gamma+\alpha(h+1)}{2 \alpha}, \ldots, \frac{\gamma+\alpha(h+1)+2 \alpha}{2 \alpha}, \\
3 / 2, h+3 / 2, \frac{\gamma+\alpha(h+1)}{2 \alpha}, \frac{1+\gamma+\alpha(h+1)}{2 \alpha}, \ldots, \frac{\gamma+\alpha(h+1)+2 \alpha-1}{2 \alpha},
\end{array}\right.
\end{aligned}
$$

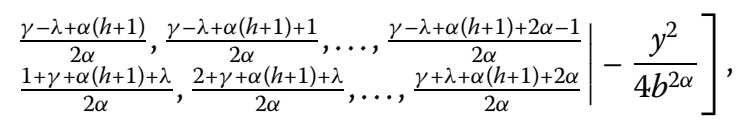

$$
\begin{aligned}
& \int_{0}^{\infty} z^{\lambda-1} A^{-\gamma} H_{h}\left(\frac{y z^{\alpha}}{A^{\alpha}}\right) d z \\
& =2^{1-(\lambda+\alpha(h+1))} b^{\lambda-\gamma}(\gamma+\alpha(h+1))\left(\frac{y}{2}\right)^{h+1} \\
& \times \frac{\Gamma(\gamma-\lambda) \Gamma(2 \lambda+2 \alpha(h+1))}{\Gamma\left(\frac{3}{2}\right) \Gamma\left(h+\frac{3}{2}\right) \Gamma(1+\lambda+\gamma+2 \alpha(h+1))} \\
& \times{ }_{1+4 \alpha} F_{2+6 \alpha}\left[\begin{array}{c}
\frac{2 \lambda+2 \alpha(h+1)}{2 \alpha}, \frac{2 \lambda+2 \alpha(h+1)+1}{2 \alpha}, \ldots, \frac{2 \lambda+2 \alpha(h+1)+2 \alpha-1}{2 \alpha}, \\
3 / 2, h+3 / 2, \frac{\gamma+\alpha(h+1)}{2 \alpha}, \frac{\gamma+\alpha(h+1)+1}{2 \alpha}, \ldots, \frac{\gamma+\alpha(h+1)+2 \alpha-1}{2 \alpha},
\end{array},\right.
\end{aligned}
$$

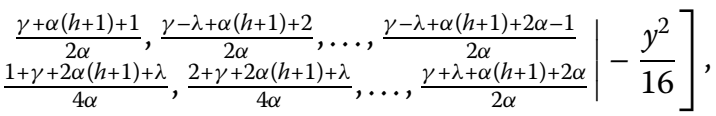

$$
\begin{aligned}
& \int_{0}^{1} z^{\lambda-1}(1-z)^{2 \gamma-1}(S)^{2 \lambda-1}(T)^{\gamma-1} H_{h}\left(y(1-z)^{2 \alpha} T^{\alpha}\right) d z \\
& =\left(\frac{2}{3}\right)^{2 \lambda}\left(\frac{y}{2}\right)^{h+1} \frac{B(\gamma+\alpha(h+1), \lambda)}{\Gamma(h+3 / 2) \Gamma(3 / 2)}
\end{aligned}
$$

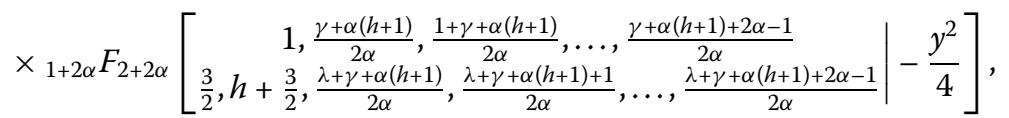

$$
\begin{aligned}
& \int_{0}^{1} z^{\lambda-1}(1-z)^{2 \gamma-1}(S)^{2 \lambda-1}(T)^{\gamma-1} H_{h}\left(y z^{\alpha} S^{2 \alpha}\right) d z \\
& =\left(\frac{2}{3}\right)^{2(\lambda+\alpha(h+1))}\left(\frac{y}{2}\right)^{h+1} \frac{B(\gamma, \lambda+\alpha(h+1))}{\Gamma(h+3 / 2) \Gamma(3 / 2)} \\
& \times{ }_{1+2 \alpha} F_{2+2 \alpha}\left[\begin{array}{c}
1, \frac{\lambda+\alpha(h+1)}{2 \alpha}, \frac{1+\lambda+\alpha(h+1)}{2 \alpha}, \\
\frac{3}{2}, h+\frac{3}{2}, \frac{\lambda+\gamma+\alpha(h+1)}{2 \alpha}, \frac{\lambda+\gamma+\alpha(h+1)+1}{2 \alpha},
\end{array}\right.
\end{aligned}
$$




$$
\left.\ldots, \frac{\lambda+\alpha(h+1)+2 \alpha-1}{2 \alpha} \mid-\left(\frac{16}{81}\right)^{\alpha} \frac{y^{2}}{4}\right] .
$$

(v) By inserting $u=1, v=2, w=1, a_{1}=1, b_{1}=(\tau+\epsilon+3) / 2, b_{2}=(\tau-\epsilon+3) / 2, l=$ $1, \mu=1, \zeta=2, h=1, \delta=\tau, m=1, k_{1}=0, A_{1}=0, A_{2}=0, B_{1}=-1, \eta=1, v=-1, \rho=1$ and $\xi=2^{\tau+1} /\{(\tau+\epsilon+1)(\tau-\epsilon+1)\}$ in Eqs. (2.1), (2.3), (2.4) and (2.6), the V-function becomes a Lommel function [5]. By using (3.1) with conditions imposed on Theorems 2.1-2.4, we, respectively, obtain

$$
\begin{aligned}
& \int_{0}^{\infty} z^{\lambda-1} A^{-\gamma} S_{\tau, \epsilon}\left(y A^{-\alpha}\right) d z \\
& =\frac{2^{1-\lambda} b^{\lambda-(\gamma+\alpha(\tau+1))} y^{\tau+1}(\gamma+\alpha(\tau+1))}{(\tau-\epsilon+1)(\tau+\epsilon+1)} \\
& \times \frac{\Gamma(2 \lambda) \Gamma(\gamma+\alpha(\tau+1)-\lambda)}{\Gamma(\lambda+\gamma+\alpha(\tau+1)+1)} \\
& \times_{1+4 \alpha} F_{2+4 \alpha}\left[\begin{array}{c}
1, \frac{1+\gamma+\alpha(\tau+1)}{2 \alpha}, \frac{2+\gamma+\alpha(\tau+1)}{2 \alpha}, \ldots, \frac{\gamma+\alpha(\tau+1)+2 \alpha}{2 \alpha}, \\
\frac{\tau-\epsilon+3}{2}, \frac{\tau+\epsilon+3}{2}, \frac{\gamma+\alpha(\tau+1)}{2 \alpha}, \frac{1+\gamma+\alpha(\tau+1)}{2 \alpha}, \ldots, \frac{\gamma+\alpha(\tau+1)+2 \alpha-1}{2 \alpha},
\end{array},\right.
\end{aligned}
$$

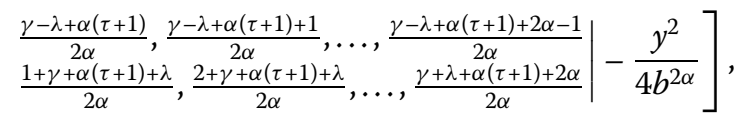

$$
\begin{aligned}
& \int_{0}^{\infty} z^{\lambda-1} A^{-\gamma} S_{\tau, \epsilon}\left(y z^{\alpha} A^{-\alpha}\right) d z \\
& =\frac{2^{1-(\lambda+\alpha(\tau+1))} b^{\lambda-\gamma} y^{\tau+1}(\gamma+\alpha(\tau+1))}{(\tau-\epsilon+1)(\tau+\epsilon+1)} \\
& \times \frac{\Gamma(2(\lambda+\alpha(\tau+1))) \Gamma(\gamma-\lambda)}{\Gamma(\lambda+\gamma+\alpha(\tau+1)+1)} \\
& \times{ }_{1+6 \alpha} F_{2+6 \alpha}\left[\begin{array}{c}
1, \frac{1+\gamma+\alpha(\tau+1)}{2 \alpha}, \frac{2+\gamma+\alpha(\tau+1)}{2 \alpha}, \ldots, \frac{\gamma+\alpha(\tau+1)+2 \alpha}{2 \alpha}, \\
\frac{\tau-\epsilon+3}{2}, \frac{\tau+\epsilon+3}{2}, \frac{\gamma+\alpha(\tau+1)}{2 \alpha}, \frac{1+\gamma+\alpha(\tau+1)}{2 \alpha}, \ldots, \frac{\gamma+\alpha(\tau+1)+2 \alpha-1}{2 \alpha},
\end{array},\right. \\
& \left.\begin{array}{rl}
\frac{2 \lambda+2 \alpha(\tau+1)}{4 \alpha} & , \frac{2 \lambda+2 \alpha(\tau+1)+1}{4 \alpha}, \ldots, \frac{2 \lambda+2 \alpha(\tau+1)+4 \alpha-1}{4 \alpha} \\
\frac{1+\gamma+2 \alpha(\tau+1)+\lambda}{4 \alpha} & \frac{2+\gamma+2 \alpha(\tau+1)+\lambda}{4 \alpha}, \ldots, \frac{\gamma+\lambda+2 \alpha(\tau+1)+4 \alpha}{4 \alpha}
\end{array} \mid-\frac{y^{2}}{4^{\alpha+1}}\right], \\
& \int_{0}^{1} z^{\lambda-1}(1-z)^{2 \gamma-1}(S)^{2 \lambda-1}(T)^{\gamma-1} S_{\tau, \epsilon}\left(y(1-z)^{2 \alpha} T^{\alpha}\right) d z \\
& =\left(\frac{2}{3}\right)^{2 \lambda} \frac{B(\gamma+\alpha(\tau+1), \lambda)}{(\tau-\epsilon+1)(\tau+\epsilon+1)} \\
& \times{ }_{1+2 \alpha} F_{2+2 \alpha}\left[\begin{array}{c}
1, \frac{\gamma+\alpha(\tau+1)}{2 \alpha}, \frac{1+\gamma+\alpha(\tau+1)}{2 \alpha}, \\
\frac{\tau-\epsilon+3}{2}, \frac{\tau+\epsilon+3}{2}, \frac{\lambda+\gamma+2 \alpha(\tau+1)}{2 \alpha}, \frac{1+\lambda+\gamma+2 \alpha(\tau+1)}{2 \alpha},
\end{array}\right. \\
& \left.\ldots, \frac{\gamma+\alpha(\tau+1)+2 \alpha-1}{2 \alpha} \mid-\frac{y^{2}}{4}\right] \\
& \int_{0}^{1} z^{\lambda-1}(1-z)^{2 \gamma-1} S^{2 \lambda-1} T^{\gamma-1} S_{\tau, \epsilon}\left(y z^{\alpha} S^{2 \alpha}\right) d z \\
& =\left(\frac{2}{3}\right)^{2(\lambda+\alpha(\tau+1))} \frac{B(\gamma, \lambda+\alpha(\tau+1))}{(\tau-\epsilon+1)(\tau+\epsilon+1)}
\end{aligned}
$$




$$
\begin{aligned}
& \times{ }_{1+2 \alpha} F_{2+2 \alpha}\left[\begin{array}{c}
1, \frac{\lambda+\alpha(\tau+1)}{2 \alpha}, \frac{1+\lambda+\alpha(\tau+1)}{2 \alpha}, \\
\frac{\tau-\epsilon+3}{2}, \frac{\tau+\epsilon+3}{2}, \frac{\lambda+\gamma+\alpha(\tau+1)}{2 \alpha}, \frac{1+\lambda+\gamma+\alpha(\tau+1)}{2 \alpha},
\end{array},\right. \\
& \left.\ldots, \frac{\lambda+\alpha(\tau+1)+2 \alpha-1}{2 \alpha} \mid-\frac{y^{2}}{4}\left(\frac{4}{9}\right)^{\alpha}\right] \text {. }
\end{aligned}
$$

(vi) By inserting $u=1, v=1, w=1, a_{1}=1, b_{1}=1, l=-2, \mu=1, \zeta=1, \delta=0, m=0, k_{1}=$ $0, A_{1}=0, B_{1}=-1, v=-1, \rho=1$ and $\xi=1 /(\Gamma(h))$ in Eqs. (2.1), (2.3), (2.4) and (2.6), the Vfunction becomes a Mittag-Leffler function [9, 23]. By using (3.1) with the understanding that the conditions of Theorems 2.1-2.4 are applied, we, respectively, obtain

$$
\begin{aligned}
& \int_{0}^{\infty} z^{\lambda-1} A^{-\gamma} E_{h, \eta}\left(\frac{y}{A^{\alpha}}\right) d z \\
& =2^{1-\lambda} b^{\lambda-\gamma}(\gamma) \frac{\Gamma(2 \lambda) \Gamma(\gamma-\lambda)}{\Gamma(h) \Gamma(\lambda+\gamma+1)} \\
& \times_{1+2 \alpha} F_{2 \alpha+\eta}\left[\begin{array}{c}
1, \frac{1+\gamma}{\alpha}, \frac{2+\gamma}{\alpha} \cdots, \frac{\gamma+\alpha}{\alpha}, \\
\frac{h}{\eta}, \frac{h+1}{\eta}, \ldots, \frac{h+\eta-1}{\eta}, \frac{\gamma}{\alpha}, \frac{1+\gamma}{\alpha} \cdots, \frac{\gamma+\alpha-1}{\alpha},
\end{array}\right.
\end{aligned}
$$

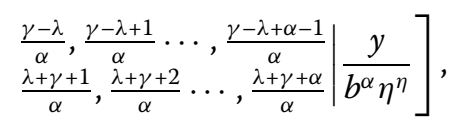

$$
\begin{aligned}
& \int_{0}^{\infty} z^{\lambda-1} A^{-\gamma} E_{h, \eta}\left(\frac{y z^{\alpha}}{A^{\alpha}}\right) d z \\
& =2^{1-\lambda} b^{\lambda-\gamma}(\gamma) \frac{\Gamma(\gamma-\lambda)}{\Gamma(h+1) \Gamma(\lambda+\gamma+1)} \\
& \times{ }_{1+3 \alpha} F_{3 \alpha+\eta}\left[\begin{array}{c}
1, \frac{1+\gamma}{\alpha}, \frac{2+\gamma}{\alpha} \cdots, \frac{\gamma+\alpha}{\alpha}, \\
\frac{h}{\eta}, \frac{h+1}{\eta}, \ldots, \frac{h+\eta}{\eta}, \frac{\gamma}{\alpha}, \frac{1+\gamma}{\alpha} \cdots, \frac{\gamma+\alpha-1}{\alpha},
\end{array}\right. \\
& \left.\begin{array}{c|c}
\frac{2 \lambda}{2 \alpha}, \frac{2 \lambda+1}{2 \alpha} \cdots, \frac{2 \lambda+2 \alpha-1}{2 \alpha} & y \\
\frac{\lambda+\gamma+1}{2 \alpha}, \frac{\lambda+\gamma+2}{2 \alpha} \cdots, \frac{\lambda+\gamma+2 \alpha}{2 \alpha} & \frac{\lambda}{2^{\alpha} \eta^{\eta}}
\end{array}\right] \\
& \int_{0}^{1} z^{\lambda-1}(1-z)^{2 \gamma-1} S^{2 \lambda-1} T^{\gamma-1} E_{h, \eta}\left(y(1-z)^{2 \alpha} T^{\alpha}\right) d z \\
& =\left(\frac{2}{3}\right)^{2 \lambda} \frac{B(\gamma, \lambda)}{\Gamma(h)} \\
& \times_{1+\alpha} F_{\alpha+\eta}\left[\begin{array}{c}
1, \frac{\gamma}{\alpha}, \frac{1+\gamma}{\alpha} \ldots, \frac{\gamma+\alpha-1}{\alpha} \\
\left.\frac{h}{\eta}, \frac{h+1}{\eta}, \ldots, \frac{h+\eta-1}{\eta}, \frac{\lambda+\gamma}{\alpha}, \frac{1+\lambda+\gamma}{\alpha} \ldots, \frac{\lambda+\gamma+\alpha-1}{\alpha} \mid \frac{y}{\eta^{\eta}}\right],
\end{array}\right. \\
& \int_{0}^{1} z^{\lambda-1}(1-z)^{2 \gamma-1} S^{2 \lambda-1} T^{\gamma-1} E_{h, \eta}\left(y z^{\alpha} S^{2 \alpha}\right) d z \\
& =\left(\frac{2}{3}\right)^{2 \lambda} \frac{B(\gamma, \lambda)}{\Gamma(h)} \\
& \times{ }_{1+\alpha} F_{\alpha+\eta}\left[\begin{array}{c}
1, \frac{\lambda}{\alpha}, \frac{1+\lambda}{\alpha} \ldots, \frac{\lambda+\alpha-1}{\alpha} \\
\left.\frac{h}{\eta}, \frac{h+1}{\eta}, \ldots, \frac{h+\eta-1}{\eta}, \frac{\lambda+\gamma}{\alpha}, \frac{1+\lambda+\gamma}{\alpha} \ldots, \frac{\lambda+\gamma+\alpha-1}{\alpha} \mid\left(\frac{4}{9}\right)^{\alpha} \frac{y}{\eta^{\eta}}\right] .
\end{array}\right.
\end{aligned}
$$

(vii) Putting $u=1, v=1, w=1, a_{1}=a, b_{1}=1, l=-2, \zeta=1, \delta=0, m=0, k_{1}=0, A_{1}=0, B_{1}=$ $0, \eta=1, v=0, \rho=0$ and $\xi=1$ in Eqs. (2.1), (2.3), (2.4) and (2.6), the V-function turns into a unified Riemann-Zeta function [7], and by using (3.1) under the conditions imposed on 
Theorems 2.1-2.4, we, respectively, get

$$
\begin{aligned}
& \int_{0}^{\infty} z^{\lambda-1} A^{-\gamma} \phi_{a}\left(y A^{-\alpha}, \mu, h\right) d z \\
&= 2^{1-\lambda} b^{\lambda-\gamma} \\
& \times \sum_{n=0}^{\infty} \frac{(a)_{n} \Gamma(\gamma-\lambda+\alpha n) \Gamma(2 \lambda) \Gamma(\gamma+\alpha n+1)}{(h+n)^{\mu} \Gamma(\gamma+\alpha n) \Gamma(\lambda+\gamma+1+\alpha n) n !}\left(\frac{y}{b^{\alpha}}\right)^{n}, \\
& \int_{0}^{\infty} z^{\lambda-1} A^{-\gamma} \phi_{a}\left(\frac{y z^{\alpha}}{A^{\alpha}}, \mu, h\right) d z \\
& \quad 2^{1-\lambda} b^{\lambda-\gamma} \frac{(a)_{n} \Gamma(\gamma-\lambda) \Gamma(2 \lambda+2 \alpha n) \Gamma(\gamma+\alpha n+1)}{(h+n)^{\mu} \Gamma(\gamma+\alpha n) \Gamma(\lambda+\gamma+1+2 \alpha n) n !}\left(\frac{y}{2}\right)^{n}, \\
& \int_{0}^{1} z^{\lambda-1}(1-z)^{2 \gamma-1} S^{2 \lambda-1} T^{\gamma-1} \phi_{a}\left(y(1-z)^{2 \alpha} T^{\alpha}\right) d z\left(\frac{2}{3}\right)^{2 \lambda} \sum_{n=0}^{\infty} \frac{(a)_{n} \Gamma(\gamma+\alpha n) \Gamma(\lambda)}{(h+n)^{\mu} \Gamma(\gamma+\lambda+\alpha n)}\left(\frac{y^{n}}{n !}\right), \\
&=\left.\frac{2}{3}\right)^{2 \lambda} \sum_{n=0}^{\infty} \frac{(a)_{n} \Gamma(\gamma) \Gamma(\lambda+\alpha n)}{(h+n)^{\mu} \Gamma(\gamma+\lambda+\alpha n)}\left(\frac{2}{3}\right)^{\alpha n} \frac{y^{n}}{n !} . \\
& \int_{0}^{1} z^{\lambda-1}(1-z)^{2 \gamma-1} S^{2 \lambda-1} T^{\gamma-1} \phi_{a}\left(y z^{\alpha} S^{2 \alpha}\right) d z
\end{aligned}
$$

(viii) Putting $u=1, v=1, w=1, a_{1}=1, b_{1}=1, l=2, \zeta=1, \delta=0, m=0, k_{1}=0, A_{1}=0, B_{1}=$ $-1, \eta=1, v=-1, \rho=1, h=1, \mu=1$, and $\xi=1$ in Eqs. (2.1), (2.3), (2.4) and (2.6), the Vfunction turns into a $e^{-z}$ function and, by using (3.1) under the conditions imposed on Theorems 2.1-2.4, respectively, we get

$$
\begin{aligned}
& \int_{0}^{\infty} z^{\lambda-1} A^{-\gamma} e^{-\left(y A^{-\alpha}\right)} d z=2^{1-\lambda} b^{\lambda-\gamma}(\gamma) \frac{\Gamma(2 \lambda) \Gamma(\gamma-\lambda)}{\Gamma(\lambda+\gamma+1)} \\
& \times{ }_{2 \alpha} F_{2 \alpha}\left[\begin{array}{l}
\frac{1+\gamma}{\alpha}, \frac{2+\gamma}{\alpha} \cdots, \frac{\gamma+\alpha}{\alpha}, \frac{\gamma-\lambda}{\alpha}, \frac{\gamma-\lambda+1}{\alpha} \cdots, \frac{\gamma-\lambda+\alpha-1}{\alpha} \\
\frac{\gamma}{\alpha}, \frac{1+\gamma}{\alpha} \cdots, \frac{\gamma+\alpha-1}{\alpha}, \frac{\lambda+\gamma+1}{\alpha}, \frac{\lambda+\gamma+2}{\alpha} \cdots, \frac{\lambda+\gamma+\alpha}{\alpha}
\end{array} \mid-y b^{-\alpha}\right], \\
& \int_{0}^{\infty} z^{\lambda-1} A^{-\gamma} e^{-\left(y z^{\alpha} A^{-\alpha}\right)} d z \\
& =2^{1-\lambda} b^{\lambda-\gamma}(\gamma) \frac{\Gamma(2 \lambda) \Gamma(\gamma-\lambda)}{\Gamma(\lambda+\gamma+1)} \\
& \times{ }_{3 \alpha} F_{3 \alpha}\left[\begin{array}{c}
\frac{1+\gamma}{\alpha}, \frac{2+\gamma}{\alpha} \ldots, \frac{\gamma+\alpha}{\alpha}, \frac{2 \lambda}{2 \alpha}, \frac{2 \lambda+1}{2 \alpha} \ldots, \frac{2 \lambda+2 \alpha-1}{2 \alpha} \\
\frac{\gamma}{\alpha}, \frac{1+\gamma}{\alpha} \cdots, \frac{\gamma+\alpha-1}{\alpha}, \frac{\lambda+\gamma+1}{2 \alpha}, \frac{\lambda+\gamma+2}{2 \alpha} \cdots, \frac{\lambda+\gamma+2 \alpha}{2 \alpha}
\end{array} \mid-\frac{y}{2^{\alpha}}\right], \\
& \int_{0}^{1} z^{\lambda-1}(1-z)^{2 \gamma-1} S^{2 \lambda-1} T^{\gamma-1} e^{-\left(y(1-z)^{2 \alpha} T^{\alpha}\right)} d z \\
& =\left(\frac{2}{3}\right)^{2 \lambda} B(\gamma, \lambda)
\end{aligned}
$$




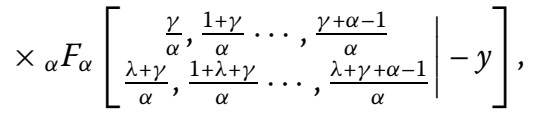

$$
\begin{aligned}
& \int_{0}^{1} z^{\lambda-1}(1-z)^{2 \gamma-1} S^{2 \lambda-1} T^{\gamma-1} e^{-\left(y z^{\alpha} S^{2 \alpha}\right)} d z \\
& =\left(\frac{2}{3}\right)^{2 \lambda} B(\gamma, \lambda) \\
& \times_{\alpha} F_{\alpha}\left[\begin{array}{c}
\frac{\lambda}{\alpha}, \frac{1+\lambda}{\alpha} \cdots, \frac{\lambda+\alpha-1}{\alpha} \\
\frac{\lambda+\gamma}{\alpha}, \frac{1+\lambda+\gamma}{\alpha} \cdots, \frac{\lambda+\gamma+\alpha-1}{\alpha} \mid
\end{array} \mid-\left(\frac{4}{9}\right)^{\alpha} y\right] .
\end{aligned}
$$

(ix) Putting $w=1, p=P, q=Q, l=2, \mu=1, \zeta=1, \delta=0, m=0, k_{u}=0, A_{u}=0, B_{1}=-1, \eta=$ $1 v=-1, \rho=1, h=1$ and $\xi=\frac{\prod_{u=1}^{P} \Gamma\left(a_{u}\right)}{\prod_{v=1}^{Q} \Gamma\left(b_{v}\right)}$ in Eqs. (2.1), (2.3), (2.4) and (2.6), the V-function turns into a Macrobert E-function [4]. Moreover, by using the result (3.1) with conditions already imposed on Theorems 2.1-2.4, respectively, we get

$$
\begin{aligned}
& \int_{0}^{\infty} z^{\lambda-1} A^{-\gamma} E\left(P,\left(a_{P}\right) ; Q,\left(b_{Q}\right) ; \frac{1}{y A^{\alpha}}\right) d z \\
& =\frac{\prod_{u=1}^{P} \Gamma\left(a_{u}\right)}{\prod_{\nu=1}^{Q} \Gamma\left(b_{v}\right)} \frac{2^{1-\lambda} b^{\lambda-\gamma} \Gamma(\gamma+1) \Gamma(2 \lambda) \Gamma(\gamma-\lambda)}{\Gamma(\gamma) \Gamma(\lambda+\gamma+1)} \\
& \times{ }_{P+2 \alpha} F_{Q+2 \alpha}\left[\begin{array}{l}
a_{1}, a_{2}, \ldots, a_{P} ; \frac{1+\gamma}{\alpha}, \frac{2+\gamma}{\alpha} \cdots, \frac{\gamma+\alpha}{\alpha}, \\
b_{1}, b_{2}, \ldots, b_{Q}, \frac{\gamma}{\alpha}, \frac{1+\gamma}{\alpha} \cdots, \frac{\gamma+\alpha-1}{\alpha},
\end{array}\right. \\
& \left.\begin{array}{c}
\frac{\gamma-\lambda}{\alpha}, \frac{\gamma-\lambda+1}{\alpha} \cdots, \frac{\gamma-\lambda+\alpha-1}{\alpha} \\
\frac{\lambda+\gamma+1}{\alpha}, \frac{\lambda+\gamma+2}{\alpha} \cdots, \frac{\lambda+\gamma+\alpha}{\alpha}
\end{array} \mid-y b^{-\alpha}\right], \\
& \int_{0}^{\infty} z^{\lambda-1} A^{-\gamma} E\left(P,\left(a_{P}\right) ; Q,\left(b_{Q}\right) ; \frac{1}{y z^{\alpha} A^{-\alpha}}\right) d z \\
& =\frac{\prod_{u=1}^{P} \Gamma\left(a_{u}\right)}{\prod_{v=1}^{Q} \Gamma\left(b_{v}\right)} \frac{2^{1-\lambda} b^{\lambda-\gamma} \Gamma(\gamma+1) \Gamma(2 \lambda) \Gamma(\gamma-\lambda)}{\Gamma(\gamma) \Gamma(\lambda+\gamma+1)} \\
& \times{ }_{P+3 \alpha} F_{Q+3 \alpha}\left[\begin{array}{l}
a_{1}, a_{2}, \ldots, a_{P} ; \frac{1+\gamma}{\alpha}, \frac{2+\gamma}{\alpha} \ldots, \frac{\gamma+\alpha}{\alpha}, \\
b_{1}, b_{2}, \ldots, b_{Q} ; \frac{\gamma}{\alpha}, \frac{1+\gamma}{\alpha} \cdots, \frac{\gamma+\alpha-1}{\alpha},
\end{array}\right. \\
& \left.\begin{array}{c}
\frac{2 \lambda}{2 \alpha}, \frac{2 \lambda+1}{2 \alpha} \cdots, \frac{2 \lambda+2 \alpha-1}{2 \alpha} \\
\frac{\lambda+\gamma+1}{2 \alpha}, \frac{\lambda+\gamma+2}{2 \alpha} \cdots, \frac{\lambda+\gamma+2 \alpha}{2 \alpha}
\end{array} \mid 2^{-\alpha} y\right] \text {, } \\
& \int_{0}^{1} z^{\lambda-1}(1-z)^{2 \gamma-1} S^{2 \lambda-1} T^{\gamma-1} E\left(P,\left(a_{P}\right) ; Q,\left(b_{Q}\right) ; \frac{1}{y(1-z)^{2 \alpha} T^{\alpha}}\right) d z \\
& =\left(\frac{2}{3}\right)^{2 \lambda} B(\gamma, \lambda) \frac{\prod_{u=1}^{P} \Gamma\left(a_{u}\right)}{\prod_{v=1}^{Q} \Gamma\left(b_{v}\right)}
\end{aligned}
$$

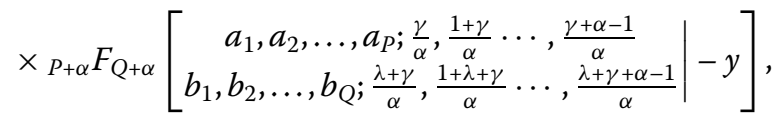

$$
\begin{aligned}
& \int_{0}^{1} z^{\lambda-1}(1-z)^{2 \gamma-1} S^{2 \lambda-1} T^{\gamma-1} E\left(P,\left(a_{P}\right) ; Q,\left(b_{Q}\right) ; \frac{1}{y z^{\alpha} S^{2 \alpha}}\right) d z \\
& =\left(\frac{2}{3}\right)^{2 \lambda} B(\gamma, \lambda) \frac{\prod_{u=1}^{P} \Gamma\left(a_{u}\right)}{\prod_{v=1}^{Q} \Gamma\left(b_{v}\right)}
\end{aligned}
$$




$$
\times{ }_{P+2 \alpha} F_{Q+2 \alpha}\left[\begin{array}{c}
a_{1}, a_{2}, \ldots, a_{P} ; \frac{2 \lambda}{2 \alpha}, \frac{1+2 \lambda}{2 \alpha} \ldots, \frac{2 \lambda+2 \alpha-1}{2 \alpha} \\
b_{1}, b_{2}, \ldots, b_{Q} ; \frac{\lambda+\gamma}{2 \alpha}, \frac{1+\lambda+\gamma}{2 \alpha} \ldots, \frac{\lambda+\gamma+2 \alpha-1}{2 \alpha}
\end{array} \mid-\left(\frac{4}{9}\right)^{\alpha} y\right]
$$

(x) Inserting $u=1, v=2, w=1, a_{1}=1, b_{1}=1, k_{1}=0, h=1 / 2, l=1, \mu=1, \zeta=2, \delta=0, m=$ $0, A_{1}=0, A_{2}=-1, B_{1}=0, \eta=1, v=-1 / 2, \rho=1$ and $\xi=1$ in Eqs. (2.1), (2.3), (2.4) and (2.6), the V-function turns into a $\cos z$ function and, by using (3.1) under the assumptions of Theorems 2.1-2.4, we, respectively, get

$$
\begin{aligned}
& \int_{0}^{\infty} z^{\lambda-1} A^{-\gamma} \cos \left(y A^{-\alpha}\right) d z \\
& =2^{1-\lambda} b^{\lambda-\gamma}(\gamma) \frac{\Gamma(2 \lambda) \Gamma(\gamma-\lambda)}{\Gamma(\lambda+\gamma+1)} \\
& \times{ }_{4 \alpha} F_{4 \alpha+1}\left[\begin{array}{c}
\frac{1+\gamma}{2 \alpha}, \frac{2+\gamma}{2 \alpha} \cdots, \frac{\gamma+2 \alpha}{2 \alpha}, \frac{\gamma-\lambda}{2 \alpha}, \frac{\gamma-\lambda+1}{2 \alpha} \cdots, \frac{\gamma-\lambda+2 \alpha-1}{2 \alpha} \\
\frac{1}{2}, \frac{\gamma}{2 \alpha}, \frac{1+\gamma}{2 \alpha} \cdots, \frac{\gamma+2 \alpha-1}{2 \alpha}, \frac{\lambda+\gamma+1}{2 \alpha}, \frac{\lambda+\gamma+2}{2 \alpha} \cdots, \frac{\lambda+\gamma+2 \alpha}{2 \alpha}
\end{array} \mid-\frac{y^{2}}{4 b^{2 \alpha}}\right], \\
& \int_{0}^{\infty} z^{\lambda-1} A^{-\gamma} \cos \left(y z^{\alpha} A^{-\alpha}\right) d z \\
& =2^{1-\lambda} b^{\lambda-\gamma}(\gamma) \frac{\Gamma(2 \lambda) \Gamma(\gamma-\lambda)}{\Gamma(\lambda+\gamma+1)} \\
& \times{ }_{6 \alpha} F_{1+6 \alpha}\left[\begin{array}{c}
\frac{1+\gamma}{2 \alpha}, \frac{2+\gamma}{2 \alpha} \cdots, \frac{\gamma+2 \alpha}{2 \alpha}, \frac{2 \lambda}{4 \alpha}, \frac{2 \lambda+1}{4 \alpha} \cdots, \frac{2 \lambda+4 \alpha-1}{4 \alpha} \\
\frac{1}{2}, \frac{\gamma}{2 \alpha}, \frac{1+\gamma}{2 \alpha} \cdots, \frac{\gamma+2 \alpha-1}{2 \alpha}, \frac{\lambda+\gamma+1}{4 \alpha}, \frac{\lambda+\gamma+2}{4 \alpha} \cdots, \frac{y^{2}}{4 \alpha}
\end{array} \mid-\frac{\lambda+4 \alpha}{4^{\alpha+1}}\right], \\
& \int_{0}^{1} z^{\lambda-1}(1-z)^{2 \gamma-1} S^{2 \lambda-1} T^{\gamma-1} \cos \left(y(1-z)^{2 \alpha} T^{\alpha}\right) d z \\
& =\left(\frac{2}{3}\right)^{2 \lambda} B(\gamma, \lambda)
\end{aligned}
$$

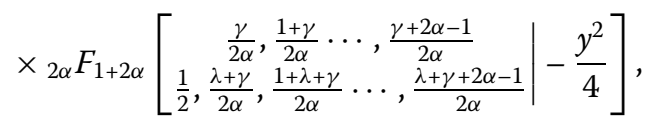

$$
\begin{aligned}
& \int_{0}^{1} z^{\lambda-1}(1-z)^{2 \gamma-1} S^{2 \lambda-1} T^{\gamma-1} \cos \left(y z^{\alpha} S^{2 \alpha}\right) d z \\
& =\left(\frac{2}{3}\right)^{2 \lambda} B(\gamma, \lambda)
\end{aligned}
$$

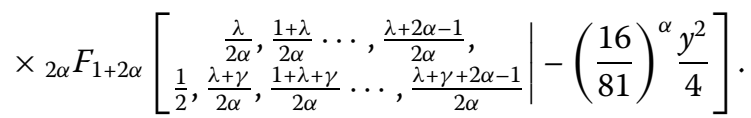

(xi) Putting $u=1, v=2, w=1, a_{1}=1, b_{1}=1, k_{1}=0, h=1 / 2, l=1, \mu=1, \zeta=2, \delta=2, m=$ $0, A_{1}=0, A_{2}=-1, B_{1}=0, \eta=1, v=-1 / 2, \rho=1$ and $\xi=1$ in Eqs. (2.1), (2.3), (2.4) and (2.6), the $\mathrm{V}$-function turns into a $\sin z$ function, then by using (3.1) under the conditions on Theorems 2.1 to 2.4 , we, respectively, get

$$
\begin{aligned}
\int_{0}^{\infty} & z^{\lambda-1} A^{-\gamma} \sin \left(y A^{-\alpha}\right) d z \\
= & 2^{1-\lambda} b^{\lambda-\gamma+\alpha}(\gamma+\alpha) \frac{\Gamma(2 \lambda) \Gamma(\gamma-\lambda+\alpha)}{\Gamma(\lambda+\gamma+\alpha+1)} \\
& \times{ }_{4 \alpha} F_{4 \alpha+1}\left[\begin{array}{c}
\frac{1+\gamma+\alpha}{2 \alpha}, \frac{2+\gamma+\alpha}{2 \alpha} \\
\frac{3}{2}, \frac{\gamma+\alpha}{2 \alpha}, \frac{1+\gamma+\alpha}{2 \alpha} \cdots, \frac{\gamma+\alpha+2 \alpha}{2 \alpha},
\end{array}\right.
\end{aligned}
$$




$$
\begin{aligned}
& \left.\begin{array}{l}
\frac{\gamma-\lambda+\alpha}{2 \alpha}, \frac{\gamma-\lambda+\alpha+1}{2 \alpha} \cdots, \frac{\gamma-\lambda+\alpha+2 \alpha-1}{2 \alpha} \\
\frac{\lambda+\gamma+\alpha+1}{2 \alpha}, \frac{\lambda+\gamma+\alpha+2}{2 \alpha} \cdots, \frac{\lambda+\gamma+\alpha+2 \alpha}{2 \alpha}
\end{array} \mid-\frac{y^{2}}{4 b^{2 \alpha}}\right], \\
& \int_{0}^{\infty} z^{\lambda-1} A^{-\gamma} \sin \left(y z^{\alpha} A^{-\alpha}\right) d z \\
& =2^{1-\lambda+\alpha} b^{\lambda-\gamma}(\gamma+\alpha) \frac{\Gamma(2 \lambda+2 \alpha) \Gamma(\gamma-\lambda)}{\Gamma(\lambda+\gamma+1+2 \alpha)} \\
& \times{ }_{6 \alpha} F_{1+6 \alpha}\left[\begin{array}{c}
\frac{1+\gamma+\alpha}{2 \alpha}, \frac{2+\gamma+\alpha}{2 \alpha} \cdots, \frac{\gamma+\alpha+2 \alpha}{2 \alpha}, \\
\frac{3}{2}, \frac{\gamma+\alpha}{2 \alpha}, \frac{1+\gamma+\alpha}{2 \alpha} \cdots, \frac{\gamma+\alpha+2 \alpha-1}{2 \alpha},
\end{array}\right. \\
& \left.\begin{array}{r}
\frac{2 \lambda+2 \alpha}{4 \alpha}, \frac{2 \lambda+2 \alpha+1}{4 \alpha} \cdots, \frac{2 \lambda+2 \alpha+4 \alpha-1}{4 \alpha} \\
\frac{\lambda+\gamma+2 \alpha+1}{4 \alpha}, \frac{\lambda+\gamma+2 \alpha+2}{4 \alpha} \cdots, \frac{\lambda+\gamma+2 \alpha+4 \alpha}{4 \alpha}
\end{array} \mid-\frac{y^{2}}{4^{\alpha+1}}\right], \\
& \int_{0}^{1} z^{\lambda-1}(1-z)^{2 \gamma-1} S^{2 \lambda-1} T^{\gamma-1} \sin \left(y(1-z)^{2 \alpha} T^{\alpha}\right) d z \\
& =\left(\frac{2}{3}\right)^{2 \lambda} B(\gamma+\alpha, \lambda) y \\
& \times{ }_{2 \alpha} F_{1+2 \alpha}\left[\begin{array}{c}
\frac{\gamma+\alpha}{2 \alpha}, \frac{1+\gamma+\alpha}{2 \alpha} \cdots, \frac{\gamma+\alpha+2 \alpha-1}{2 \alpha} \\
\frac{3}{2}, \frac{\lambda+\gamma+\alpha}{2 \alpha}, \frac{1+\lambda+\gamma+\alpha}{2 \alpha} \cdots, \frac{\lambda^{2}}{2 \alpha}
\end{array} \mid-\frac{y^{2}}{4}\right], \\
& \int_{0}^{1} z^{\lambda-1}(1-z)^{2 \gamma-1} S^{2 \lambda-1} T^{\gamma-1} \sin \left(y z^{\alpha} S^{2 \alpha}\right) d z \\
& =\left(\frac{2}{3}\right)^{2 \lambda+2 \alpha} B(\gamma, \lambda+\alpha) y \\
& \times{ }_{2 \alpha} F_{1+2 \alpha}\left[\begin{array}{c}
\frac{\lambda+\alpha}{2 \alpha}, \frac{1+\lambda+\alpha}{2 \alpha} \cdots, \frac{\lambda+\alpha+2 \alpha-1}{2 \alpha} \\
\frac{3}{2}, \frac{\lambda+\gamma+\alpha}{2 \alpha}, \frac{1+\lambda+\gamma+\alpha}{2 \alpha} \cdots, \frac{\lambda+\gamma+\alpha+2 \alpha-1}{2 \alpha}
\end{array} \mid-\left(\frac{16}{81}\right)^{\alpha} \frac{y^{2}}{4}\right] .
\end{aligned}
$$

\section{Concluding remarks}

In the present paper, we have investigated some new integral formulas involving the Vfunction, which are expressed in terms of suitable special functions. Also, we have easily seen that the exponential function, the Mittag-Leffler function, the Lommel function, the Struve function, the Wright generalized Bessel function, the Bessel function and the generalized hypergeometric function are special cases of the V-function. Therefore, the results presented in this paper are easily converted in terms of various special functions after some suitable parametric replacements. The V-functions are associated with a wide range of problems in diverse areas of mathematical physics, for example, neutron physics, plasma physics and radio physics. So the results presented in this paper may be applicable in the theory of mathematical physics as well.

Acknowledgements

The authors are very thankful to the reviewers for valuable comments and suggestions to improve the paper.

Funding

Not available.

Availability of data and materials

Not applicable. 
Authors' contributions

The authors contributed equally and significantly in writing this paper. All authors read and approved the final manuscript.

\section{Author details}

${ }^{1}$ Science and Humanities Department, L.D. College of Engineering, 380015, Ahmedabad, India. ${ }^{2}$ Department of Physics and Applied Sciences, Al-Balqa Applied University, Amman, Jordan. ${ }^{3}$ Department of Mathematics, Wollo University, 1145 , Dessie, Ethiopia.

\section{Publisher's Note}

Springer Nature remains neutral with regard to jurisdictional claims in published maps and institutional affiliations.

Received: 30 June 2020 Accepted: 30 September 2020 Published online: 07 October 2020

\section{References}

1. Agarwal, P., Jain, S., Agarwal, S., Nagpal, M.: On a new class of integrals involving Bessel functions of the first kind. Commun. Numer. Anal. 2014, Article ID cna-00216 (2014)

2. Choi, J., Agarwal, P.: Certain unified integrals associated with Bessel functions. Bound. Value Probl. 2013, 95 (2013)

3. Choi, J., Agarwal, P., Mathur, S., Purohit, S.D.: Certain new integral formulas involving the generalized Bessel functions. Bull. Korean Math. Soc. 51, 995-1003 (2014)

4. Erdelyi, A., Magnus, W., Oberhettinger, F., Tricomi, F.G.: Higher Transcendental Functions, Vol. I. McGraw-Hill, New York (1953)

5. Erdelyi, A., Magnus, W., Oberhettinger, F., Tricomi, F.G.: Higher Transcendental Functions, Vol. II. McGraw-Hill, New York (1953)

6. Exton, H.: Handbook of Hypergeometric Integrals. Ellis Horwood, Chichester (1978)

7. Goyal, S.P., Laddha, R.K.: On the generalized Riemann zeta function and the generalized Lambert JS transform. Ganita Sandesh 11(2), 99-108 (1997)

8. Haq, S., Nisar, K.S., Khan, A.H., Suthar, D.L.: Certain integral transforms of the generalized Lommel-Wright function CUBO 21(1), 49-60 (2019)

9. Humbert, P., Agarwal, R.P.: Sur la fonction de Mittag-Leffler et quelques unesdeses generalisations. Bull. Sci. Math. 77(2), 180-185 (1953)

10. Kilbas, A.A., Srivastava, H.M., Trujillo, J.J.: Theory and Applications of Fractional Differential Equations. Elsevier, Amsterdam (2006)

11. Kilbas, A.A., Sebastian, N.: Generalized fractional integration of Bessel function of the first kind. Integ. Trans. Spl. Funct. 19(12), 869-883 (2008)

12. Kiryakova, V.S.: Generalized Fractional Calculus and Applications. Pitman Research Notes in Mathematics Series. Longman, Harlow (1993)

13. Kritika, A.R., Purohit, S.D.: Mathematical model for anomalous subdiffusion using conformable operator. Chaos Solitons Fractals 140, 110199 (2020)

14. Kumar, V.: A general class of functions and N-fractional calculus. J. Rajasthan Acad. Phys. Sci. 11(3), 223-230 (2012)

15. Kumar, V.: N-fractional calculus of general class of functions and Fox's H-function. Proc. Natl. Acad. Sci. Sect. A Phys. Sci. 83(3), 271-277 (2013)

16. Kumar, V.: The Euler transform of V-function. Afr. Math. 29, 23-27 (2018)

17. Kumar, V.: On Riemann-Liouville fractional integral operator of a general class of functions. J. Rajasthan Acad. Phys. Sci. 18(3 \& 4), 193-200 (2019)

18. Lavoie, J.L., Trottier, G.: On the sum of certain Appells series. Ganita 201, 31-32 (1969)

19. Mathai, A.M., Saxena, R.K., Haubold, H.J.: The H-Function Theory and Application. Springer, New York (2010)

20. Menaria, N., Purohit, S.D., Parmar, R.K.: On a new class of integrals involving generalized Mittag-Leffler function. Surv. Math. Appl. 11, 1-9 (2016)

21. Miller, K.S., Ross, B.: An Introduction to the Fractional Calculus and Fractional Differential Equations. A Wiley-Interscience Publication. Wiley, New York (1993)

22. Mishra, A.M., Bohra, M., Purohit, S.D., Kumar, D., Singh, J.: Integrals involving generalized multi-index Bessel functions and general class of polynomials. Math. Eng. Sci. Aerosp. 11(2), 415-424 (2020)

23. Mittag-Leffler, G.: Sur la nouvelle fonction $E_{\alpha}(x)$. C.R. Acad. Sci. Paris 137, 554-558 (1903)

24. Nisar, K.S., Agarwal, P., Jain, S.: Some unified integrals associated with Bessel-Struve kernel function (2016) arXiv:1602.01496v1 [math.CA]

25. Nisar, K.S., Mondal, S.R.: Certain unified integral formulas involving the generalized modified k-Bessel function of first kind. Commun. Korean Math. Soc. 32, 47-53 (2017)

26. Nisar, K.S., Suthar, D.L., Purohit, S.D., Aldhaifallah, M.: Some unified integral associated with the generalized Struve function. Proc. Jangjeon Math. Soc. 20(2), 261-267 (2017)

27. Nisar, K.S., Suthar, D.L., Purohit, S.D., Amsalu, H.: Unified integrals involving product of multivariable polynomials and generalized Bessel functions. Bol. Soc. Parana. Mat. 38(6), 73-83 (2020)

28. Oberhettinger, F.: Tables of Mellin Transforms. Springer, New York (1974)

29. Samko, S.G., Kilbas, A.A., Marichev, O.I.: Fractional Integrals and Derivatives: Theory and Applications. Gordon \& Breach, Yverdon (1993)

30. Sharma, J.B., Sharma, K.K., Purohit, S.D., Atangana, A.: Hybrid watermarking algorithm using finite radon and fractional Fourier transform. Fundam. Inform. 151, 523-543 (2017)

31. Suthar, D.L., Amsalu, H.: Generalized fractional integral operators involving Mittag-Leffler function. Abstr. Appl. Anal. 2018, Article ID 7034124 (2018)

32. Suthar, D.L., Purohit, S.D., Nisar, K.S.: Certain integrals associated with generalized hypergeometric functions. Acta Univ. Apulensis 55, 105-112 (2018) 
33. Suthar, D.L., Purohit, S.D., Nisar, K.S.: Integral transforms of the Galue type Struve function. TWMS J. Appl. Eng. Math. 8(1), 114-121 (2018)

34. Suthar, D.L., Andualem, M., Debalkie, B.: A study on generalized multivariable Mittag-Leffler function via generalized fractional calculus operators. J. Math. 2019, Article ID 9864737 (2019). https://doi.org/10.1155/2019/9864737

35. Al-Omari, S.: Some estimate of a generalized Bessel-Struve transform on certain space of generalized functions. Ukr. Math. J. 69(9), 1155-1165 (2017)

36. Al-Omari, S.: A study on a class of modified Bessel-type integrals in a Fréchet space of Boehmians. Bol. Soc. Parana. Mat. 38(4), 145-156 (2020)

37. Khan, N., Usman, T., Aman, M., Al-Omari, S., Choi, J.: Integral transforms and probability distributions involving generalized hyper geometric function. Georgian Math. J. (2021, to appear)

38. Agarwal, P., Jain, S., Ahmad, B., Al-Omari, S.: Certain recent fractional integral inequalities associated with the hypergeometric operators. J. King Saud Univ., Sci. 4(17), 1-10 (2015)

\section{Submit your manuscript to a SpringerOpen ${ }^{\circ}$} journal and benefit from:

- Convenient online submission

- Rigorous peer review

- Open access: articles freely available online

- High visibility within the field

- Retaining the copyright to your article

Submit your next manuscript at $\gg$ springeropen.com 